\title{
Intoxicação espontânea por Ipomoea carnea subsp. fistulosa (Convolvulaceae) em bovinos no Pantanal Matogrossense ${ }^{1}$
}

\author{
Nadia A.B. Antoniassi ${ }^{2}$, Eduardo V. Ferreira² ${ }^{2}$ Carlos E.P. dos Santos ${ }^{2}$, Laura P. de \\ Arruda $^{3}$, João Losano E. Campos ${ }^{4}$, Luciano Nakazato ${ }^{2}$ e Edson M. Colodel ${ }^{2 *}$
}

\begin{abstract}
Antoniassi N.A.B., Ferreira E.V., Santos C.E.P., Campos J.L.E., Nakazato L. \& Colodel E.M. 2007. [Spontaneous Ipomoea carnea subsp. fistulosa (Convolvulaceae) poisoning of cattle in the Brazilian Pantanal.] Intoxicação espontânea por Ipomoea carnea subsp. fistulosa (Convolvulaceae) em bovinos no Pantanal Matogrossense. Pesquisa Veterinária Brasileira 27(10):415-418. Departamento de Clínica Médica Veterinária, Faculdade de Agronomia e Medicina Veterinária, Universidade Federal de Mato Grosso, Cuiabá, MT 78068-900, Brazil. E-mail: moleta@ufmt.br

A spontaneous Ipomoea carnea subsp. fistulosa (canudo, algodoeiro) poisoning of cattle in the county of Poconé, Brazilian Pantanal, is reported. The investigation began after 12 cattle had died from a flock of 500 animals maintained in an extensive area intensely infested by $I$. carnea subsp. fistulosa with scarce availability of other fodder plants. The deaths occurred from June to September of 2006. Clinical signs were loss of weight and neurological deficits with hypermetry and incoordination. No significant gross lesions were observed at postmortem examination of one bovine. Histological changes comprised widespread cytoplasmic vacuolation of neurons, cells of the thyroid, kidney and pancreas. Cattle with similar clinical picture, that had been removed from the area invaded by I. carnea subsp. fistulosa and placed into areas with native and Brachiaria sp. pasture, recovered clinically within 15 days.
\end{abstract}

INDEX TERMS: Poisonous plants, Ipomoea carnea subsp. fistulosa, plant poisoning, storage diseases, cattle.

RESUMO.- Relata-se a intoxicação espontânea por Ipomoea carnea subsp. fistulosa (canudo, algodoeiro) em bovinos no Pantanal Matogrossense. As investigações iniciaram após a morte de 12 bovinos, de um rebanho de 500 animais, criados em uma extensa área intensamente infestada por I. carnea subsp. fistulosa com escassa disponibilidade de outra forragem. As mortes ocorreram entres os meses de junho e setembro de 2006. O quadro clínico foi caracterizado por emagrecimento e sinais neurológicos com dificuldade locomotora. Um bovino foi necropsiado sem que se observassem alterações macroscópicas significativas. Histologicamente havia tumefação e vacuolização celular, em neurônios, células acinares pancreáticas, tubulares renais e foliculares da tireóide. Bovinos com quadro clínico similar fo-

\footnotetext{
${ }^{1}$ Recebido em 9 de maio de 2007.

Aceito para publicação em 24 de maio de 2007.

2 Depto Clínica Médica Veterinária, Faculdade de Agronomia e Medicina Veterinária (FAMEV), Universidade Federal de Mato Grosso (UFMT), Av. Fernando Correa da Costa s/n, Bairro Coxipó, Cuiabá, MT 78068-000, Brasil. *Autor para correspondência: moleta@ufmt.br

3 Programa de Pós-Graduação em Ciências Veterinárias, FAMEV, UFMT.

${ }^{4}$ Medico Veterinário Autônomo, Fazenda Ipiranga, Rod. Transpantaneira, Km 10, Poconé, MT 78175-000, Brasil.
}

ram retirados da área invadida por $I$. carnea subsp. fistulosa e colocadas em áreas com pastagem nativa e de Brachiaria sp. e apresentaram melhora clínica após período de 15 dias.

TERMOS DE INDEXAÇÃO: Plantas tóxicas, Ipomoea carnea subsp. fistulosa, intoxicação por planta, doença de armazenamento, bovinos, Pantanal Matogrossense.

\section{INTRODUÇÃO}

Ipomoea carnea Jacq. subsp. fistulosa (Martius ex Choisy), sin. Ipomoea fistulosa Mart., família Convolvulaceae, é uma planta arbustiva ereta, no pantanal conhecida como "algodão-do-Pantanal”, "algodão-bravo" ou "algodoeiro” (Frey 1995). É denominada também "canudo" ou "capa-bode" na região Nordeste (Tokarnia et al. 2000). Esta planta é resistente à seca e se propaga rapidamente nos campos inundáveis do Pantanal Matogrossense, competindo com as forrageiras nativas, usadas para alimentação de gado local (Haase 1999).

I. carnea subsp. fistulosa, assim como Sida carpinifolia (Driemeier et al. 2000, Colodel et al. 2002), Turbina cordata (Datas et al. 2006), Ipomoea sericophylla, Ipomoea riedelii (Barbosa et al. 2006), Solanum fastigiatum (Rech et al. 2006), Phalaris angusta (Gava et al. 1999) são plantas descritas no Brasil como 
causa de doenças de depósito lisossomal em ruminantes. Essas doenças são caracterizadas pelo depósito de substratos em lisossomos devido a alterações de atividade de hidrolases ácidas (Jolly \& Walkley 1997).

No Brasil, I. carnea subsp. fistulosa possui maior importância como planta tóxica na região Nordeste, sobretudo no vale do Rio São Francisco e no sul do Piauí, onde a planta permanece verde durante todo o período de estiagem (Tokarnia et al. 2000). A intoxicação por $I$. subsp. fistulosa ocorre em condições de fome, quando os animais ingerem a planta em períodos de escassez de pastagem, se alimentando quase que exclusivamente da planta durante semanas (Tokarnia et al. 1960, 2000). No Pantanal Matogrossense, local em que a planta é abundante, não foram documentados casos de intoxicação por I. carnea subsp. fistulosa.

Os principais sinais clínicos são de origem nervosa, observando-se incoordenação com dismetria e instabilidade principalmente no trem posterior (Tokarnia et al. 1960, 2000, Damir et al. 1987). Estes sinais são precipitados e evidenciados quando os animais estão sob condição de estresse. São observados também emagrecimento progressivo, lassidão e pêlos ásperos (Tokarnia et al. 1960, 2000, Balogh et al. 1999). Na necropsia não são observadas alterações significativas e histologicamente pode-se visualizar tumefação e fina vacuolização de neurônios em diversas áreas do sistema nervoso central, ocasionalmente de células da glia e formações de esferóides axonais. Vacuolizações citoplasmáticas ocorrem também em hepatócitos, células acinares do pâncreas e macrófagos do baço e gânglios linfáticos (Balogh et al. 1999, Tokarnia et al. 2000).

O presente trabalho tem por objetivo descrever clinica e patologicamente a intoxicação espontânea por Ipomoea carnea em bovinos no Pantanal Matogrossense.

\section{MATERIAL E MÉTODOS}

Dados epidemiológicos e clínicos da intoxicação por Ipomoea carnea subsp. fistulosa em bovinos foram obtidos junto ao proprietário e veterinários responsáveis pelo rebanho, durante visitas a propriedade em área do Pantanal Matogrossense, no Município de Poconé, Mato Grosso.

Dois bovinos, nelores, 4 anos de idade, fêmeas, com quadro clínico neurológico foram examinados e um foi eutanasiado e necropsiado. Deste bovino o encéfalo, medula espinhal e fragmentos de fígado, rim, coração, pulmão, linfonodos, baço, músculo esquelético, intestino, gânglio trigeminal, foram coletados e fixados em solução de formol a $10 \%$, processados rotineiramente, corados por hematoxilina e eosina (HE) e analisados microscopicamente. $\mathrm{O}$ outro bovino com o mesmo quadro clínico foi retirado, junto com o rebanho, da área infestada por Ipomoea carnea subsp. fistulosa, colocado em área com predomínio de capim-mimoso, braquiária e andropogon, e acompanhado clinicamente em intervalos semanais.

\section{Histórico clínico}

\section{RESULTADOS}

Durante o período entre os meses de junho até o mês de setembro de 2006, em um rebanho bovino, com aproximadamente 500 cabeças da raça nelore, criados extensivamente no Pantanal Matogrossense, 12 bovinos foram encontrados mortos durante os rodeios que eram feitos com periodicidade semanal. Alguns destes bovinos foram anteriormente observados magros e com andar lento. Um período atípico de estiagem durante os primeiros meses do ano seguido de período chuvoso em março e abril, na região do Pantanal de Poconé, foi atribuído como causa do fraco desenvolvimento de forrageiras como "capim-mimoso" (Axonopus sp.) e Andropogon sp. e intenso desenvolvimento vegetativo de Ipomoea carnea subsp. fistulosa, sendo que em algumas áreas esta planta se constituía como a única fonte de vegetação disponível para alimentação dos bovinos.

Durante a visita no local de pastoreio dos bovinos, em meados do mês de setembro de 2006, se observou a escassez de pastagens e de invasão difusa de I. carnea subsp. fistulosa na área (Fig.1). As partes aéreas da planta apresentavam intensamente consumidas e notando-se que alguns bovinos a pastoreavam com avidez, durante o período em que foram observadas. Fraco escore corporal e pelagem arrepiada eram achados comuns em vários bovinos do rebanho.

\section{Quadro clínico}

Os bovinos examinados estavam isolados do rebanho, acentuadamente magros. Alterações neurológicas eram ob-
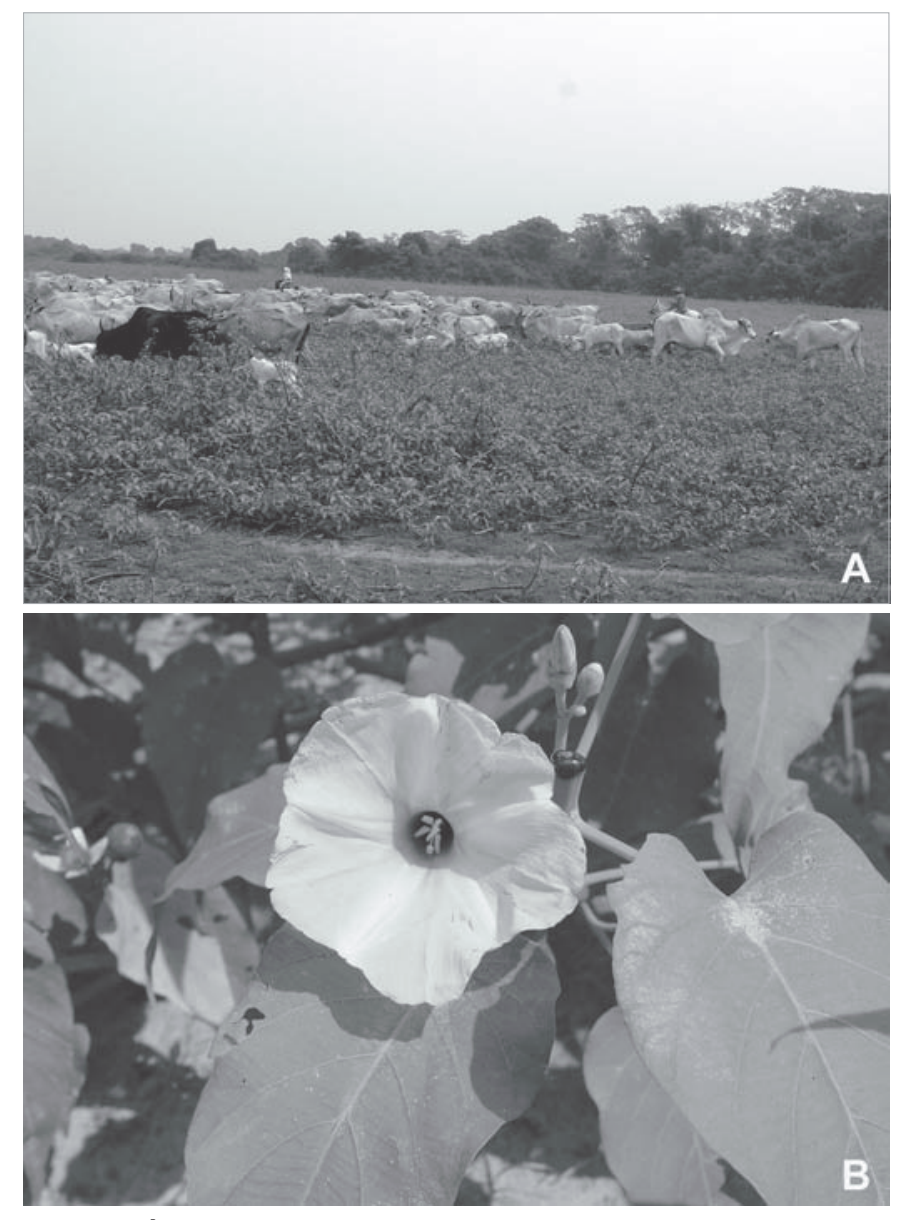

Fig.1. (A) Área de pastoreio de bovinos, no Pantanal Matogrossense, invadida por Ipomoea carnea subsp. fistulosa, Poconé, MT; (B) planta com inflorescência, setembro de 2006. 


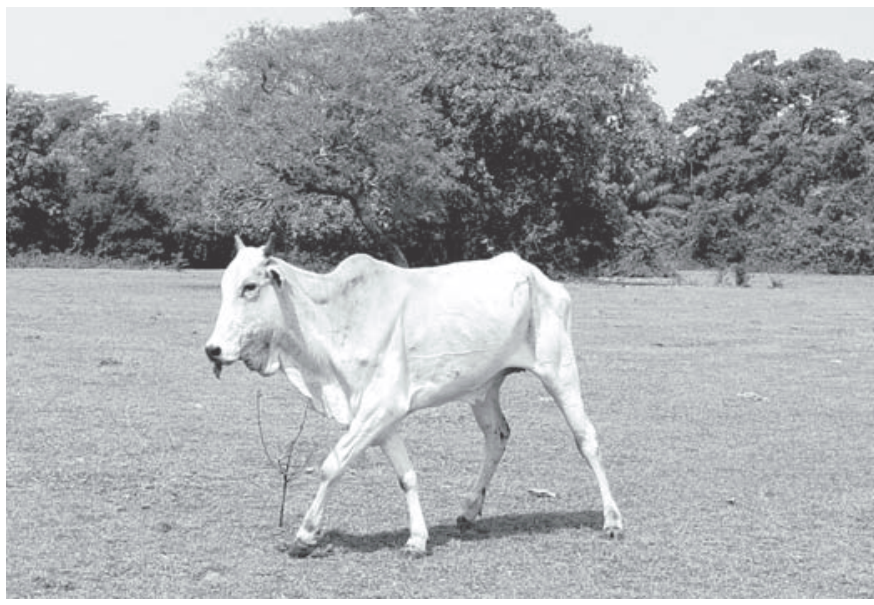

Fig.2. Bovino com dismetria associada ao consumo de Ipomoea carnea subsp. fistulosa no Pantanal Matogrossense. Este bovino permanecia por longos períodos com alimento na boca sem movimentos de mastigação.

servadas quando os bovinos eram movimentados, exibindo incoordenação motora com ataxia e dismetria principalmente de membros posteriores além de tremores musculares principalmente de cabeça e pescoço e retenção de alimento na boca (Fig.2). Notava-se também que os bovinos estavam agressivos a aproximação, mas demonstravam fraqueza e quedas durante as investidas. Um dos bovinos foi retirado da área com I. carnea subsp. fistulosa, junto com o rebanho, colocado em área com pastagem nativa (capim mimoso, Andropogon sp. e Brachiaria sp.) e apresentou melhora clínica após um período de 15 dias.

\section{Achado patológicos}

Não foram observadas alterações significativas durante a necropsia. Microscopicamente no sistema nervoso central observaram-se em diversas áreas neurônios com citoplasma espumoso, principalmente em neurônios da medula oblonga (Fig.3), mesencéfalo e tálamo. Moderada e difusa vacuolização

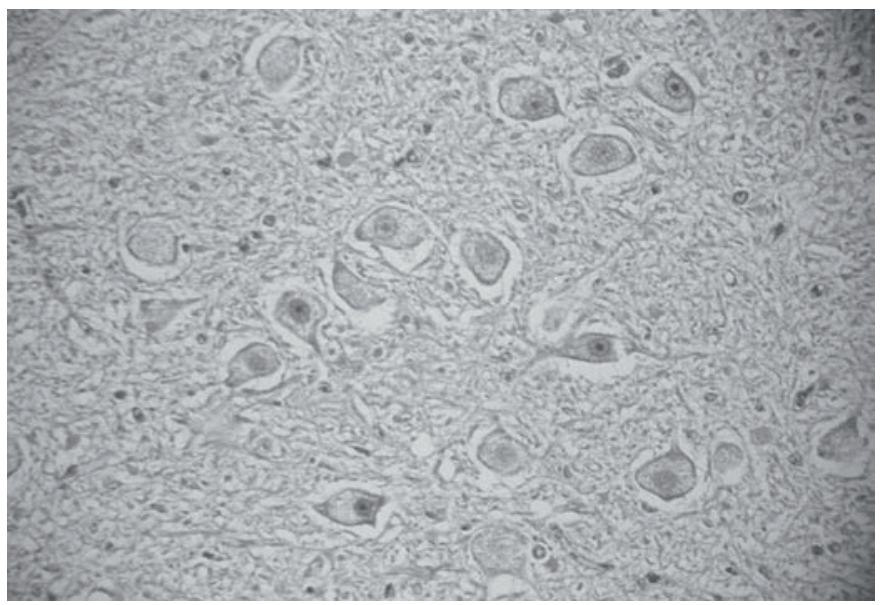

Fig.3. Vacuolização do citoplasma de neurônios da medula oblonga, na intoxicação natural por Ipomoea carnea subsp. fistulosa em bovino. HE, obj.10x (dsx 2431).

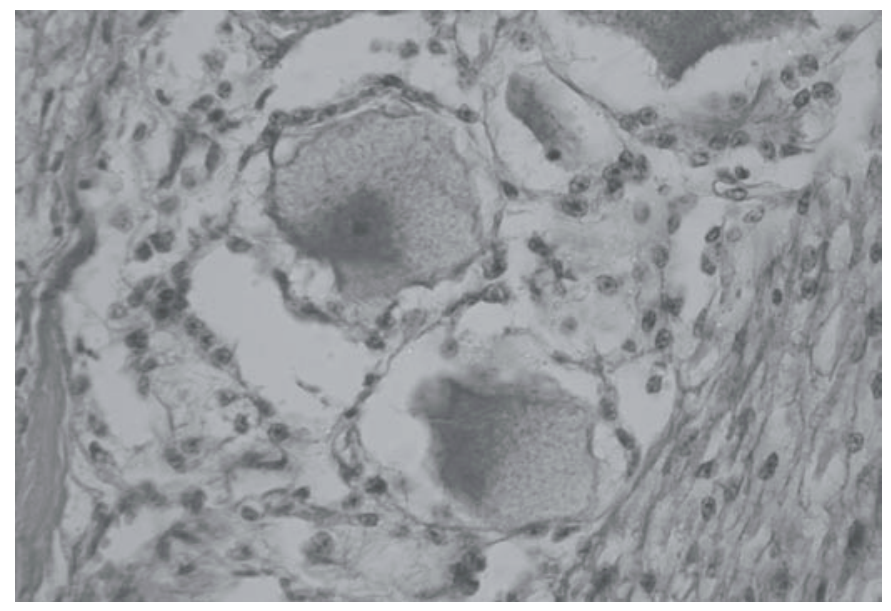

Fig.4. Tumefação e vacuolização de neurônios do gânglio trigeminal de bovino naturalmente intoxicado por Ipomoea carnea subsp. fistulosa. HE, obj.40x (2335).

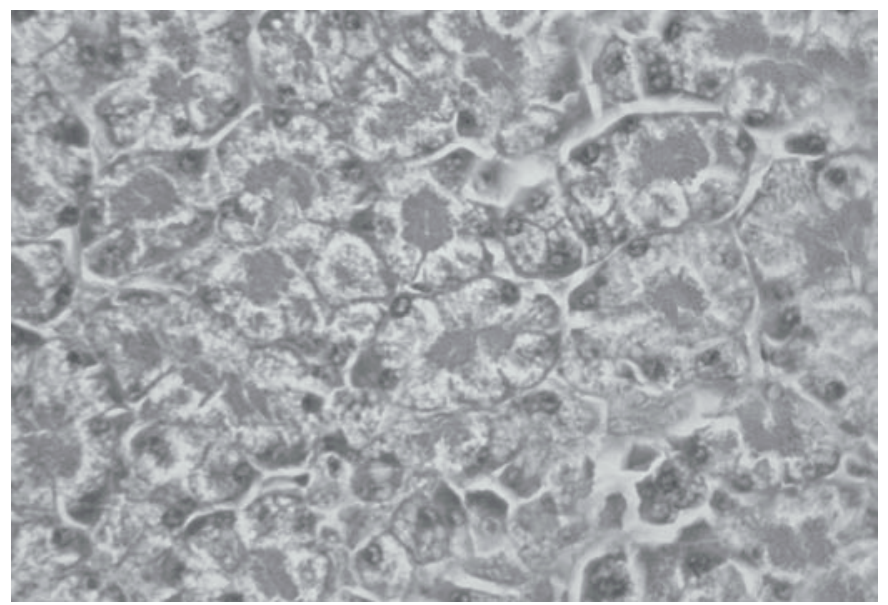

Fig.5. Difusa tumefação e vacuolização do epitélio acinar pancreático em bovino intoxicado naturalmente por Ipomoea carnea subsp. fistulosa. HE, obj.20x (2437).

foi observada em neurônios do Gânglio trigêmeo (Fig.4). Alterações menos evidentes foram observadas em neurônios de Purkinje, do córtex cerebral e da medula espinhal. Havia também variável vacuolização em hepatócitos, células acinares pancreáticas (Fig.5), células foliculares da tireóide e células tubulares renais.

\section{DISCUSSÃO}

O diagnóstico de intoxicação por Ipomoea carnea subsp. fistulosa foi baseado no histórico, sinais clínicos e principalmente nos achados histológicos. Não há evidências para afirmar que todas as mortes neste rebanho, durante o período de seca no Pantanal, tenham relação com a ingestão dessa planta, porém as observações de quadro clínico compatível durante rodeios, da melhora de bovinos clinicamente afetados e da diminuição das mortes após mudança do rebanho para área com disponibilidade de forragens, não infestada com I. carnea subsp. fistulosa, são evidências da importância desta planta como causa das alterações clínicas, para este rebanho de bovinos. 
Os sinais clínicos relatados neste caso são similares aos observados em outros trabalhos de intoxicação por I. carnea subsp. fistulosa em ruminantes (Balogh et al. 1999, Tokarnia et al. 2000, Schumaher-Henrique 2003). Estes sinais refletem principalmente as alterações relacionadas à vacuolização de neurônios. A vacuolização celular, em caprinos intoxicados por I. carnea subsp. fistulosa, foi associada ao depósito de oligossacarídeos que contêm alfa-manosil, alfa-D-glicosamina e resíduo de ácido N-acetilneuramínico (Armién, 2000). Estes achados histológicos caracterizam os quadros de doença de depósito lisossomal. Alcalóides como swainsonina, 2-epi-lentiginosina, calisteginas B1, B2, B3, e C1 foram isolados de I. carnea subsp. fistulosa e causam alterações na atividade das enzimas lisossomal alfa-glicosidase, alfamanosidase e beta-manosidase (Molyneux et al. 1995, Haraguchi et al. 2003, Hueza et al. 2005). A swainsonina é encontrada em outras plantas que foram relacionadas como causa de intoxicação em ruminantes causando fina vacuolização celular, como Swainsona canescens (Coolegate et al. 1979), Astragalus sp. (Molyneux \& James, 1982), Oxytropis sp. (Ralphs et al. 2002), Sida carpinifolia (Colodel et al. 2002), Tubina cordata (Dantas et al. 2006), Ipomoea sericophylla e Ipomoea riedelii (Barbosa et al. 2006). Para o diferencial desses quadros clínicos observações epidemiológicas como distribuição geográfica e sazonalidade devem ser consideradas. Solanum fastigiatum, relatado no Sul do Brasil como causa de doença de depósito, cursa com fina vacuolização celular limitada aos neurônios de Purkinje no cerebelo (Riet-Correa et al. 1983, Rach et al. 2006).

A forma tradicional de criação extensiva de bovinos no Pantanal Matogrossense, com extensas áreas de pastoreio, rebanhos grandes e em sistema de pastejo contínuo, inviabiliza o isolamento de áreas invadidas com I. carnea subsp. fistulosa como forma de controle da doença. Os proprietários da região relatam com frequiência não estimada, a manifestação de sinais clínicos em bovinos e a recuperação quando são retirados para áreas livres da planta. Em caprinos, após apresentarem sinais clínicos acentuados e por longos períodos, não foi notado recuperação, mesmo deixando de ingerir a planta (Tokarnia et al. 1960).

Alguns métodos químicos e físicos de controle de $I$. carnea subsp. fistulosa são descritos (Afonso \& Pott 2001). Haase (1999) observou o padrão sazonal do desenvolvimento vegetativo da planta, crescendo vigorosamente durante o início da estação chuvosa e diminuindo acentuadamente quando a área está alagada, preconizando controle mecânico com roçadas em áreas invadidas, o mais próximo possível do início das chuvas, minimizando a infestação da área de pastoreio por I. carnea subsp. fistulosa.

\section{REFERÊNCIAS}

Afonso E. \& Pott A. 2001. Plantas no Pantanal tóxicas para bovinos. Embrapa Gado de Corte, Campo Grande, MT. 51p.

Armién A.G. 2000. Vergleichende klinische und morphologische Untersuchungen zur spontanen und experimentellen Vergiftung durch Ipomoea fistulosa (Convolvulaceae) bei Ziegen. Dissertation, Institut für Veterinär-Pathologie der Justus-Liebig-Universität. Giessen, Alemanha. $211 \mathrm{p}$.

Barbosa R.C., Riet-Correa F., Medeiros R.M., Lima E.F., Barros S.S., Gimeno E.J., Molyneux R.J. \& Gardner D.R. 2006 Intoxication by Ipomoea sericophylla and Ipomoea riedelii in goats in the state of Paraiba, Northeastern Brazil. Toxicon 47:371-379.
Balogh K.K.I.M., Dimande A.P., Lugt J.J., Molyneux R.J., Naudé T.W. \& Welman W.G.A. 1999. Lisosomal storage disease induced by Ipomoea carnea in goats in Mozambique. J. Vet. Diagn. Invest. 11:266-273.

Colegate S.M., Dorling P.R. \& Huxtable C.R. 1979. A spectroscopic investigation of swainsonina: an alfa-mannosidase inhibitor isolated from Swainsona canescens. Aust. J. Chem. 32:2257-2264.

Colodel E.M., Driemeier D., Loretti A.P., Gimeno E.J., Traverso S.D., Seitz A.L. \& Zlotowski P. 2002. Aspectos clínicos e patológicos da intoxicação por Sida carpinifolia (Malvaceae) em caprinos no Rio Grande do Sul. Pesq. Vet. Bras. 22:51-57.

Colodel E.M., Gardner D.R., Zlotwski P. \& Driemeier D. 2002. Identification of swainsonine as a glycoside inhibitor responsible for Sida carpinifolia poisoning. Vet. Hum. Toxicol. 44(3):177-178

Damir H.A., Adam S.E. \& Tartour G. 1987. The effects of Ipomoea carnea on goats and sheep. Vet. Hum. Toxicol. 29:316-9.

Driemeier D., Colodel E.M., Gimeno E.J. \& Barros S.S. 2000. Lysosomal storage disease caused by Sida carpinifolia poisoning in goats. Vet. Pathol. 37:153159.

Dantas A.F., Riet-Correa F., Gardner D.R., Medeiros R.M., Barros S.S., Anjos B.L. \& Lucena R.B. 2006. Swainsonine-induced lysosomal storage disease in goats caused by the ingestion of Turbina cordata in Northeastern Brazil. Toxicon. (In publication)

Frey R. 1995. Ipomoea carnea ssp. fistulosa (Martius ex-Choisy) Austin: Taxonomy, biology and ecology reviewed and inquired. Tropical Ecology 36:21-48

Gava A., Sousa R.S., de Deus M.S., Pilati C., Cristani J., Mori A. \& Neves D.S. 1999. Phalaris angusta (Gramineae) como causa de enfermidade neurológica em bovinos no Estado de Santa Catarina. Pesq. Vet. Bras.19:35-38.

Haase R. 1999. Seasonal growth of algodão-bravo (Ipomoea carnea spp. fistulosa). Pesq. Agropec. Bras. 34:159-163.

Haraguchi M., Gorniak S.L., Ikeda K., Minami Y., Kato A., Watson A.A., Nash R.J., Molyneux R.J. \& Asano N. 2003. Alkaloidal components in the poisonous plant, Ipomoea carnea (Convolvulaceae). J. Agric. Food. Chem. 51:4995-5000.

Hueza I.M., Guerra J.L., Haraguchi M., Naoki A. \& Gorniak S.L. 2005. The role of alkaloids in Ipomoea carnea toxicosis: a study in rats. Exp. Toxicol. Pathol. 57:53-58.

Jolly R.D. \& Walkley S.U. 1997. Lysosomal storage diseases of animals: an essay in comparative pathology. Vet. Pathol. 34:527-548.

Molyneux R.J., McKenzie R.A., O’Sullivan B.M. \& Elbein A.D. 1995. Identification of the glycosidase inhibitors swainsonine and calystegine B2 in Weir vine (Ipomoea sp. Q6 [aff. calobra]) and correlation with toxicity. J. Nat. Prod. 58:878-86.

Molyneux R.J. \& James L.F. 1982. Loco intoxication: indolizidine alkaloids of spotted locoweed (Astragalus lentiginosus). Science 216:190-191.

Rech R.R., Rissi D.R., Rodrigues A., Pierezan F., Piazer J.V.M., Kommers G.D. \& Barros C.S.L. 2006. Intoxicação por Solanum fastigiatum (Solanaceae) em bovinos: epidemiologia, sinais clínicos e morfometria das lesões cerebelares. Pesq. Vet. Bras. 26:183-189.

Ralphs M.H., Welsh S.L. \& Gardner D.R. 2002. Distribution of locoweed toxin swainsonine in populations of Oxytropis lambertii. J. Chem. Ecol. 28:701707.

Riet-Correa F., Méndez M.C., Schild A.L., Summers B.A. \& Oliveira J.A. 1983. Intoxication by Solanum fastigiatum var. fastigiatum as a cause of cerebellar degeneration of cattle. Cornell Vet. 73:240-256.

Schumaher-Henrique B., Gorniak S.L., Dagli M.L. \& Spinosa H.S. 2003. The clinical, biochemical, haematological and pathological effects of long-term administration of Ipomoea carnea to growing goats. Vet. Res. Commun. 27:311-319.

Tokarnia C.H., Döbereiner J. \& Canella C.F.C. 1960. Estudo experimental sobre a toxidez do "canudo" (Ipomoea fistulosa Mart.) em ruminantes. Arqs Inst. Biol. Animal, Rio de J., 3:59-71.

Tokarnia C.H., Döbereiner J. \& Peixoto P.V. 2000. Plantas Tóxicas do Brasil. Editora Helianthus, Rio de Janeiro. 310p. 